\title{
The harmonic oscillator in quantum mechanics: A third way
}

\author{
F. Marsiglio \\ Department of Physics, University of Alberta, Edmonton, Alberta, Canada, T6G 2J1
}

(Dated: December 30, 2008)

\begin{abstract}
Courses on undergraduate quantum mechanics usually focus on solutions of the Schrödinger equation for several simple one-dimensional examples. When the notion of a Hilbert space is introduced only academic examples are used, such as the matrix representation of Dirac's raising and lowering operators or the angular momentum operators. We introduce some of the same one-dimensional examples as matrix diagonalization problems, with a basis that consists of the infinite set of square well eigenfunctions. Undergraduate students are well equipped to handle such problems in familiar contexts. We pay special attention to the one-dimensional harmonic oscillator. This paper should equip students to obtain the low lying bound states of any one-dimensional short range potential.
\end{abstract}

PACS numbers:

\section{INTRODUCTION}

A standard undergraduate text in quantum mechanics ${ }^{1}$ devotes most of its attention to analytical solutions of the Schrödinger equation. Some texts 2,3 coordinate problem solving in quantum mechanics with numerical solutions. However, the focus remains the solution of the Schrödinger equation.

A typical introductory course also includes a treatment of the more formal part of quantum mechanics, with topics such as vector spaces and the matrix representation. This part is usually more abstract. Examples that are often used in this part are two state systems and matrix representations of angular momentum. Alternatively, an actual (say, $3 \times 3$ ) matrix is given, and students are asked to use matrix algebra to mimic a solution of a quantum mechanical problem. These examples often strike students as artificial. The main part of such a course appears to students to be the solution of various differential equations, and the formal part of the course often appears to be superfluous.

Numerical solutions of the time-independent Schrödinger equation have been discussed in various contexts (see, for example, Ref. 4). These solutions utilize numerical techniques for solving a differential equation. Even in the rare instance that an actual basis set is used,,$\frac{5}{}$ it is used to determine the time evolution for the time-dependent Schrödinger equation. Many quantum mechanical research problems that are amenable to solution (for example, the behavior of electrons on a small lattice) are solved using matrix mechanics, 6 The purpose of this note is to bring attention to a wide variety of time-independent problems that can be tackled through matrix mechanics at an introductory level.

In the following section we set up the infinite square well problem. The solution to this problem constitutes the (infinite) basis set with which we can tackle any single particle potential problem that can be embedded in the infinite square well. In Sec. III we illustrate the matrix solutions for the harmonic oscillator potential. This example is chosen because the students will have already encountered it using the algebraic method (series solution) and Dirac's operator method. The former method tends to leave students struggling with the mathematics, and the latter method leaves many students awestruck. The method proposed here uses matrix algebra, which is a more familiar mathematical method.

We also illustrate wave functions, which can be readily calculated from the eigenvector solutions. We have been unable to find problems of this nature in the literature. One reference illustrates a solution to the threedimensional harmonic oscillator bounded by two impenetrable walls, but does so following the standard route of differential equations, though in cylindrical coordinates $\underline{7}$

The matrix methodology discussed here is at the heart of many applications in physics. For example, vibrations in solids 8 are treated using springs that obey harmonic oscillator potentials. Bose condensation in alkali gases 9 utilizes a harmonic trap, and the conventional theory of superconductivity uses electron-ion interactions with harmonic oscillators 10 Calculations of a single electron interacting with vibrating ions (that is, a polaron) that use matrix diagonalization can be found in Ref. 11 .

The convergence criterion for the harmonic oscillator potential is related to energy scales, and the number of basis states required to accurately represent these solutions is dictated by the energies of the basis states. In Sec. IV we briefly discuss some other familiar interactions, such as the finite square well and the delta function potential. In some of these cases length scales also play an important role, which leads to a discussion of the convergence criterion connected with capturing the short length scales in the potential.

This paper is meant to be sufficiently self-contained and should serve to enhance the undergraduate curriculum in quantum mechanics, and equip undergraduates to solve a variety of useful problems.

\section{THE FORMALISM}

A typical introductory course begins with onedimensional problems, including the free particle, the in- 
finite square well, and the harmonic oscillator potential. The free particle has certain pathologies associated with the normalization of the stationary states. For this reason we prefer to discuss the infinite square well, in which a particle is confined to the space enclosed by the walls of the well. The harmonic oscillator is also amenable to analytical solution. Unlike the infinite square well the solutions (Hermite polynomials enveloped by Gaussians) are unfamiliar to the novice student, and, as far as a typical student is concerned, might as well be numerical.

For these reasons we focus on the infinite square well, which is defined as

$$
V_{\mathrm{i} n f}(x)= \begin{cases}0 & \text { if } 0<x<a \\ \infty & \text { otherwise }\end{cases}
$$

The Hamiltonian is given by

$$
H_{0}=-\frac{\hbar^{2}}{2 m} \frac{d^{2}}{d x^{2}}+V_{\mathrm{i} n f}(x)
$$

where $m$ is the mass of the particle. The eigenstates are well known:

$$
\psi_{n}(x)= \begin{cases}\sqrt{\frac{2}{a}} \sin \left(\frac{n \pi x}{a}\right) & \text { if } 0<x<a \\ 0 & \text { otherwise }\end{cases}
$$

with eigenvalues,

$$
E_{n}^{(0)}=\frac{n^{2} \pi^{2} \hbar^{2}}{2 m a^{2}} \equiv n^{2} E_{1}^{(0)} .
$$

The quantum number $n=1,2,3, \ldots$ takes on a positive integer value. Students are generally unaware of perturbation theory at this stage, but reasons for the superscript (0) can be readily explained.

Although we have presented the well-known solutions for the infinite square well problem, the emphasis in the preceding paragraphs should be on the formulation and presentation of a convenient and familiar set of basis functions. We are now prepared to tackle a variety of problems, represented by $H=H_{0}+V(x)$, where $H_{0}$ is as in Eq. (2), and $V(x)$ is any potential in the domain $0<x<a$. We proceed as in most textbooks, except now we have the specific basis set Eq. (3) in mind.

We start with the Schrodinger equation in ket notation:

$$
\left(H_{0}+V\right)|\psi\rangle=E|\psi\rangle
$$

If we use the general expansion of a wave function in terms of a complete set of basis states,

$$
|\psi\rangle=\sum_{m=1}^{\infty} c_{m}\left|\psi_{m}\right\rangle
$$

we obtain

$$
\sum_{m=1}^{\infty} c_{m}\left(H_{0}+V\right)\left|\psi_{m}\right\rangle=E \sum_{m=1}^{\infty} c_{m}\left|\psi_{m}\right\rangle .
$$

By taking the inner product of (7) with the bra $\left\langle\psi_{n}\right|$, we obtain the matrix equation:

$$
\sum_{m=1}^{\infty} H_{n m} c_{m}=E c_{n}
$$

where

$$
\begin{aligned}
& H_{n m}=\left\langle\psi_{n}\left|\left(H_{0}+V\right)\right| \psi_{m}\right\rangle= \\
& \delta_{n m} E_{n}^{(0)}+\frac{2}{a} \int_{0}^{a} d x \sin \left(\frac{n \pi x}{a}\right) V(x) \sin \left(\frac{m \pi x}{a}\right)
\end{aligned}
$$

is the Hamiltonian matrix; in the second term we have used the eigenstates of $H_{0}$ as the basis set. As usual, $\delta_{n m}$ is the Kronecker delta function.

Equation (8) along with Eq. (9) allows us to study a variety of interesting problems, albeit in the space determined by the infinite square well. Note that for numerical solutions of Eq. (8) we need to truncate the sum and hence the number of eigenstates used to describe the solutions. We denote the maximum basis set number by $N$.

\section{THE HARMONIC OSCILLATOR}

We imbed the harmonic oscillator potential, $V_{\mathrm{HO}}=$ $m \omega^{2} x^{2} / 2$, in the infinite square well. In the following all units of distance will be in terms of the square well width $a$, and all units of energy will be in terms of the (unperturbed) ground state, $E_{1}^{(0)}$. We will use lower case letters to denote dimensionless energies. The potential $V_{\mathrm{HO}}$ can be written in terms of the infinite square well length and energy scales as

$$
v_{\mathrm{HO}}=\frac{V_{\mathrm{HO}}}{E_{1}^{(0)}}=\frac{\pi^{2}}{4}\left(\frac{\hbar \omega}{E_{1}^{(0)}}\right)^{2}\left(\frac{x}{a}-\frac{1}{2}\right)^{2},
$$

so that the dimensionless parameter $\hbar \omega / E_{1}^{(0)}$ determines the stiffness of the harmonic oscillator potential. We expect that for low energy states (say, the ground state), the solution should be identical to that of the harmonic potential alone, because the wave function will be sufficiently restricted to the central region of the harmonic oscillator potential so that it will not "feel" the walls of the infinite square well. High energy states will not be well described by the harmonic oscillator results, because they will be primarily governed by the infinite square well.

We first use Eq. (9) with the potential given by Eq. (10). The result is

$$
\begin{aligned}
& h_{n m} \equiv H_{n m} / E_{1}^{(0)} \\
= & \delta_{n m}\left\{n^{2}+\frac{\pi^{2}}{48}\left(\frac{\hbar \omega}{E_{1}^{(0)}}\right)^{2}\left(1-\frac{6}{(\pi n)^{2}}\right)\right\} \\
+ & \left(1-\delta_{n m}\right)\left(\frac{\hbar \omega}{E_{1}^{(0)}}\right)^{2} g_{n m},
\end{aligned}
$$


where

$$
g_{n m}=\left(\frac{(-1)^{n+m}+1}{4}\right)\left(\frac{1}{(n-m)^{2}}-\frac{1}{(n+m)^{2}}\right) .
$$

Note that the $g_{n m}$ remain of order unity close to the diagonal, but for large $n$ the diagonal elements grow as $n^{2}$, so the off-diagonal elements become negligible in comparison.

Equation (11) can be evaluated up to some cutoff for a given $\hbar \omega / E_{1}^{(0)}$; these form the elements of a matrix to be fed into an eigenvalue/eigenvector solver (see for example, Numerical Recipes ${ }^{12}$ ) or in software packages such as Matlab, Mathematica, or Maple.

A typical result is shown in Fig. 1, The low lying states are well described by the harmonic oscillator eigenvalues. The harmonic oscillator potential becomes truncated by the infinite square well at an energy given by the value of the potential at $x=a$ (see Eq. 10): $E_{\text {cross }} / E_{1}^{(0)}=$ $\left(\pi^{2} / 16\right)\left(\hbar \omega / E_{1}^{(0)}\right)^{2}$. For this example, $\hbar \omega / E_{1}^{(0)}=50$, so this crossover occurs at $E_{\text {cross }} / E_{1}^{(0)} \approx 1550$, which is approximately where the numerical results in Fig. 1 begin to deviate from the analytical harmonic oscillator result. For higher energies the numerical results begin to resemble those of the infinite square well, albeit with an added constant, $\bar{v}_{0}$. Inspection of Eq. (9) (see also Eq. (11)) shows that this constant is the average of the harmonic potential.

What happens if the harmonic potential is considerably less stiff? If, for example, $\hbar \omega / E_{1}^{(0)}=1$, then none of the eigenstates will describe harmonic oscillator states; rather they all will be minor perturbations of the infinite square well eigenstates. Nonetheless, to describe such an oscillator is still possible, simply by choosing a much wider infinite square well.

How well is the wave function reproduced by this truncated diagonalization technique? We use Eq. (6) and obtain

$$
\psi(x)=\langle x \mid \psi\rangle=\sum_{m=1}^{\infty} c_{m} \sqrt{\frac{2}{a}} \sin \frac{m \pi x}{a},
$$

where the $c_{m}$ are the components of the eigenvectors in the basis of the infinite square well eigenfunctions. For numerical results we use Eq. (13) with the coefficients obtained from the eigenvectors determined by the matrix diagonalization. For the harmonic oscillator the results are known analytically. For example, in dimensionless units the ground state wave function is

$$
\psi_{H O}(x)=\left(\frac{\pi}{2 a^{2}} \frac{\hbar \omega}{E_{1}^{(0)}}\right)^{1 / 4} \exp \left[-\frac{\pi^{2}}{4} \frac{\hbar \omega}{E_{1}^{(0)}}\left(\frac{x}{a}-\frac{1}{2}\right)^{2}\right] .
$$

In Fig. 2] we plot the numerical results superimposed with the analytical ones. The agreement is excellent (beyond what can be discerned on a graph with this scale). We can also check the components $c_{m}$ (see Eq. (13)) directly, because they are determined through diagonaliza-

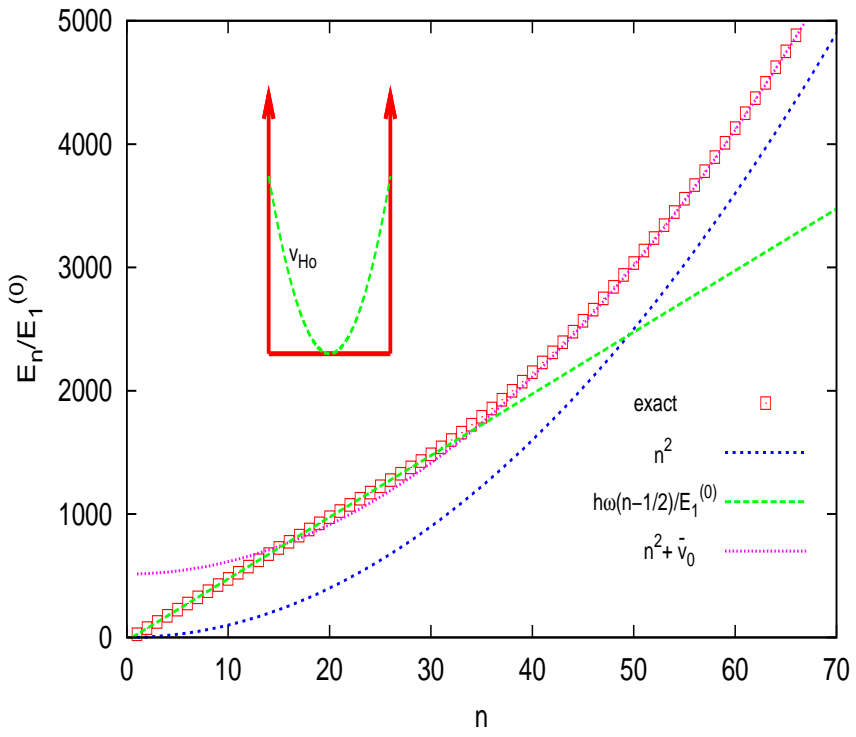

FIG. 1: (color online) Eigenvalues obtained by the exact diagonalization of the harmonic oscillator embedded in an infinite square well (shown as an inset). The symbols denote the numerically converged result, obtained with a truncation $N=400$. We also show the known analytical result for a harmonic oscillator, $E_{\mathrm{HO}}=\hbar \omega(n-1 / 2)$ (we use an enumeration that starts with one (not zero) consistent with the enumeration of the infinite square well eigenvalues). The agreement is excellent for low-lying eigenvalues. For eigenvalues that are comparable to or exceed the crossover energy the analytical results for the infinite square well, suitably modified to take approximate account of the harmonic oscillator potential, are extremely accurate (dotted curve). We used $\hbar \omega / E_{1}^{(0)}=50$.

tion, and compare them with the expected Fourier components of the Gaussian wave function. For example, for the ground state, these are

$$
c_{n}= \begin{cases}i^{(n-1)}\left(\frac{32}{\pi} \frac{E_{1}^{(0)}}{\hbar \omega}\right) \exp \left[-n^{2} E_{1}^{(0)} / \hbar \omega\right] & \text { for } n \text { odd } \\ 0 & \text { for } n \text { even }\end{cases}
$$

The exponential decay with quantum number $n$ helps to explain why so few components are required to obtain an accurate representation of the Gaussian ground state wave function. When we checked the numerically determined coefficients against those given by Eq. (15) (not shown), we found excellent agreement.

The important point is that low lying states for any potential can be obtained to any desired precision by setting up the matrix in Eq. (9). Analytical evaluations of the integrals are not even required. $\stackrel{13}{\underline{3}}$ For the harmonic oscillator this method requires the least amount of mathematics. More importantly, it serves to illustrate in a very concrete and elementary way the matrix formalism that is often presented only abstractly in textbooks. 


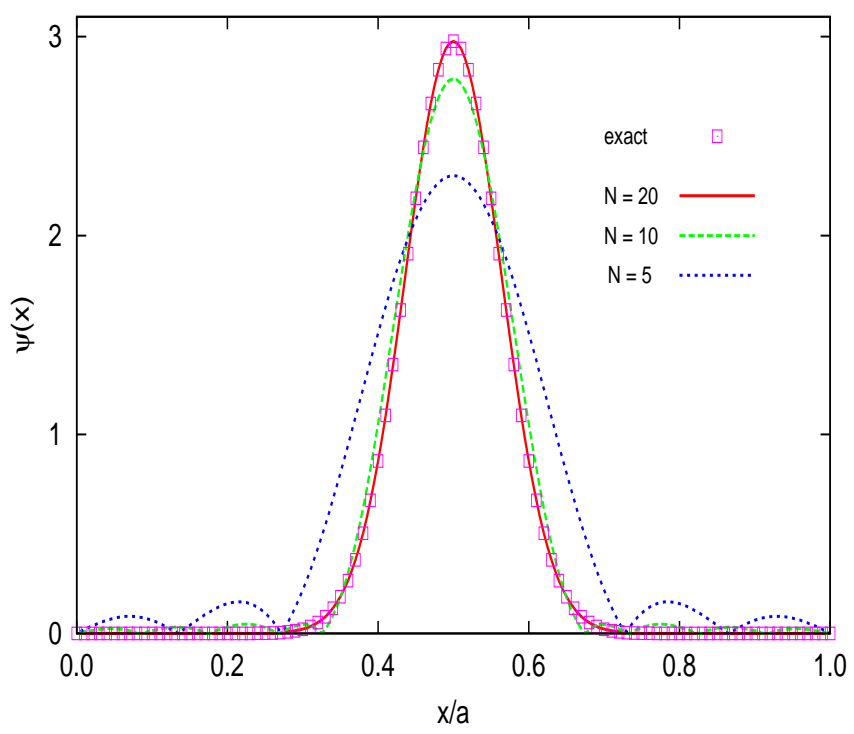

FIG. 2: (color online) The ground state of the harmonic oscillator using the analytical result, Eq. (14) (symbols), along with numerical results, obtained from matrix diagonalization, with various truncation values. Convergence is achieved for $N=20$. We used $\hbar \omega / E_{1}^{(0)}=50$.

\section{RESULTS FOR SOME STANDARD ONE-DIMENSIONAL POTENTIALS}

There are a variety of even simpler one-dimensional potentials, and this section illustrates how results from matrix mechanics can make contact with known analytical solutions.

Consider the potential of the form,

$$
V_{0}(x)= \begin{cases}0 & \text { if } 0<x<b_{0} \\ V_{0} & \text { if } b_{0}<x<b_{1} \\ 0 & \text { if } b_{1}<x<a \\ \infty & \text { otherwise }\end{cases}
$$

This potential represents a generic barrier (well) for $V_{0}$ greater (less) than zero. This case can be solved analytically although the algebra is tedious. The units are as before, and we let $\rho_{0} \equiv b_{0} / a, \rho_{1} \equiv b_{1} / a$, and $\rho=\rho_{1}-\rho_{0}$.

Evaluation of Eq. (9) yields

$$
\begin{aligned}
& h_{n m} \equiv H_{n m} / E_{1}^{(0)} \\
= & \delta_{n m}\left\{n^{2}+v_{0}\left[\rho-\frac{\sin 2 \pi n \rho_{1}-\sin 2 \pi n \rho_{0}}{2 \pi n}\right]\right\} \\
+ & v_{0}\left(1-\delta_{n m}\right)\left\{\frac{\sin (n-m) \pi \rho_{1}-\sin (n-m) \pi \rho_{0}}{\pi(n-m)}\right. \\
& \left.\left.\quad-\frac{\sin (n+m) \pi \rho_{1}-\sin (n+m) \pi \rho_{0}}{\pi(n+m)}\right\}\right\}(17)
\end{aligned}
$$

As with the harmonic oscillator, Eq. (17) can be evaluated up to some cutoff, and the resulting matrix problem

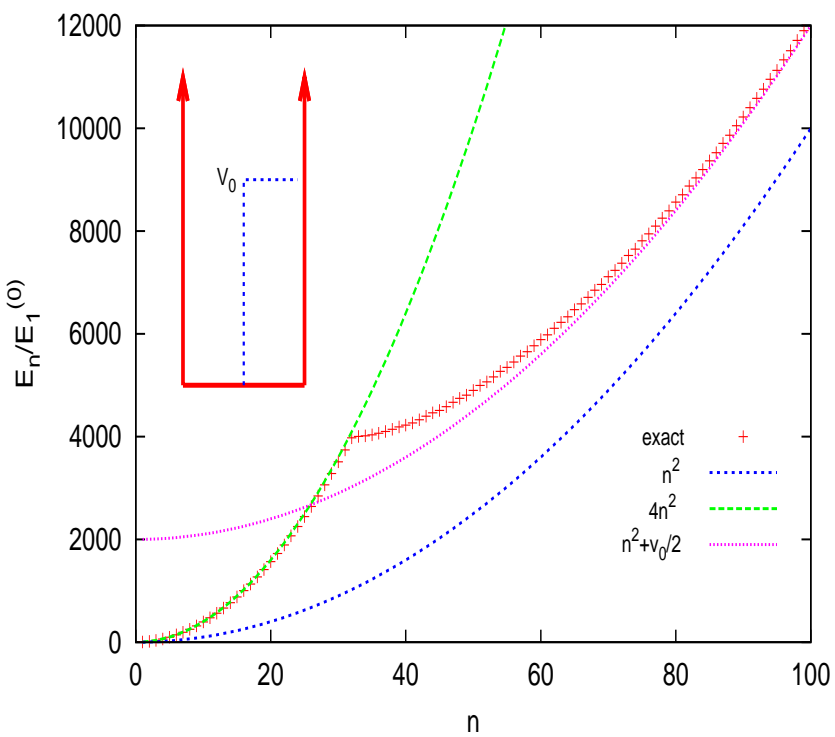

FIG. 3: (color online) Eigenvalues plotted as a function of quantum number $n$ for the potential shown in the inset. Symbols denote numerically determined eigenvalues (for a matrix truncation $N=400$, but a much smaller value yields identical results). The dashed curve illustrates the analytical result expected for an infinite square well of width $a / 2$. The shortdashed curve shows the same result for a well of width $a$. These analytical results agree with the numerical results for low and high eigenvalues, respectively. Agreement for high eigenvalues improves significantly when a shift of $v_{0} / 2$ (dotted curve) is included to account approximately (as far as the high energy states are concerned) for the presence of the step potential. We used $v_{0}=4000$; the value of $N$ is not important if it exceeds the maximum quantum number displayed by about an additional 10 .

represented by Eq. (8) can be substituted into an eigenvalue/eigenvector solver. We now consider some special cases.

\section{A. Step potential}

One of the simplest potentials is that of a potential barrier as depicted in the insert of Fig. 3. The expression in Eq. (17) applies, with $b_{0}=a / 2$ and $b_{1}=a$. Figure 3 shows the eigenvalues as a function of the quantum number $n$. The symbols show the numerically obtained eigenvalues, and the dashed curve shows the result expected from Eq. (4), with width $a / 2$ - hence the extra factor of 4. For low-lying eigenvalues the agreement is very good. In other words, the particle finds itself in an essentially infinite square well of width $a / 2$. However, for sufficiently high energy states the particle should act like it is in an infinite square well of width $a$. We have also plotted the result expected for an infinite square well of width $a$, given by the short-dashed curve. Some accommodation should be made for the fact that a step in the 
potential exists at low energies. (Imagine a state with (dimensionless) energy more than 100000 on the scale of Fig. 3 - the step with height $v_{0}=4000$ would appear to be a small blip.) The average of this potential is just the product of the height and the width, so the dotted curve corresponds to the dashed curve shifted by $v_{0} / 2$. There are a number of states whose energies are close to $v_{0}$, where the eigenvalues are not simply related to those of an infinite square well. Beyond quantum number $n=80$ the eigenvalues, with a simple accounting for the step $v_{0} / 2$, agree with those of an infinite square well. This example also illustrates how the numerical solution converges to known analytical results at low and high energies.

\section{B. Various square wells}

Consider a square well with arbitrary width less than $a$, with $V_{0}<0$. This interaction can be solved analytically, although the algebra is tedious. For $V_{0}<0$ there will exist states that are bound within the inner finite well. Insofar as these states don't "feel" the walls of the infinite square well at $x / a=0,1$, these energies and their eigenfunctions should be given by the simple analytical solution found in textbooks. The ground state is obtained by finding the lowest positive solution to the equation, $\frac{1}{\underline{n}}$

$$
\tan \frac{\pi u}{2}=\sqrt{\left(\frac{u_{0}}{u}\right)-1}
$$

where $b \equiv b_{1}-b_{0}, u \equiv \frac{b}{a} \sqrt{\frac{E-V_{0}}{E_{1}^{(0)}}}$, and $u_{0} \equiv \frac{b}{a} \sqrt{-\frac{V_{0}}{E_{1}^{(0)}}}$ (for $V_{0}<0$ and $V_{0}<E<0$ ). In the dimensionless units used in Sec. IVA $u=\rho \sqrt{e-v_{0}}$, and similarly for $u_{0}$.

For the finite square well problem analytical results are provided by the solution to Eq. (18), 14 and numerical results are obtained by diagonalizing the $N \times N$ matrix with the elements given by Eq. (17). Specifically, we use $b_{0}=a / 2-b / 2, b_{1}=a / 2+b / 2$, and $V_{0}=V_{00} a / b$. For a fixed value of $V_{00}$, this choice of potential (see Fig. 4) allows a study of the eigenenergies and eigenstates of square wells with width $b(0<b<a)$, keeping the product $b V_{0}$ fixed. The limit $b \rightarrow 0$ describes an attractive delta function potential in the middle of the infinite square well, which is treated analytically in the Appendix. The other limit, $b \rightarrow a$, lowers the baseline of the infinite square well by an amount $V_{0}$, so that all the eigenvalues are shifted: $E_{n} / E_{1}^{(0)} \rightarrow n^{2}-V_{00} / E_{1}^{(0)}$ (for $b=a$ only).

Results for the ground state energy as a function of the finite square well width $b$ are shown in Fig. 4 for various values of the cutoff $N$ (see Fig. 4(b)). The results are insensitive to the cutoff for $b \approx a$, while for low values of $b$, there is considerable variation. The inset in Fig. 4(a) clarifies the situation for $b \approx a$. The result converges very rapidly (just the diagonal element is exactly correct for
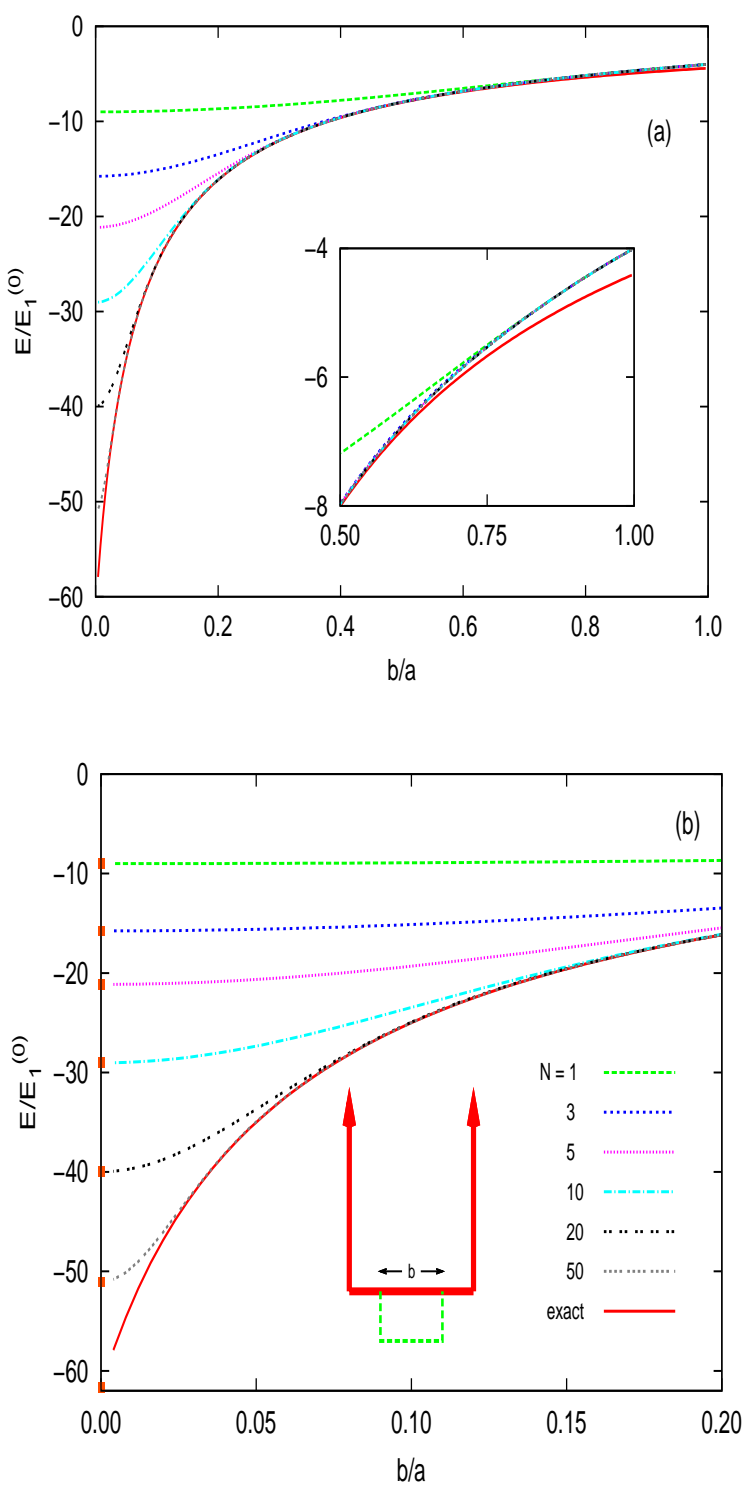

FIG. 4: (color online) Ground state energy as a function of finite square well width. The finite square well is imbedded symmetrically within the infinite square well, as shown in the insert. We use $V_{00}=-5 E_{1}^{(0)}$, and vary $V_{0}=V_{00} a / b$ as we vary $b$. Part (a) shows that convergence as a function of truncation (see legend in part (b)) is slow for small $b / a$. The inset shows the situation at large $b / a$, where the results have converged, but not to the analytical result for a square well in an infinite space, but rather, as expected, to the trivial result expected for a slightly deeper infinite square well. In part (b) we expand the low $b / a$ portion of (a), and also show analytical results for the $\delta$-function potential, shown as symbols (see Appendix). Convergence is slow because of the need to capture very small length scales. 


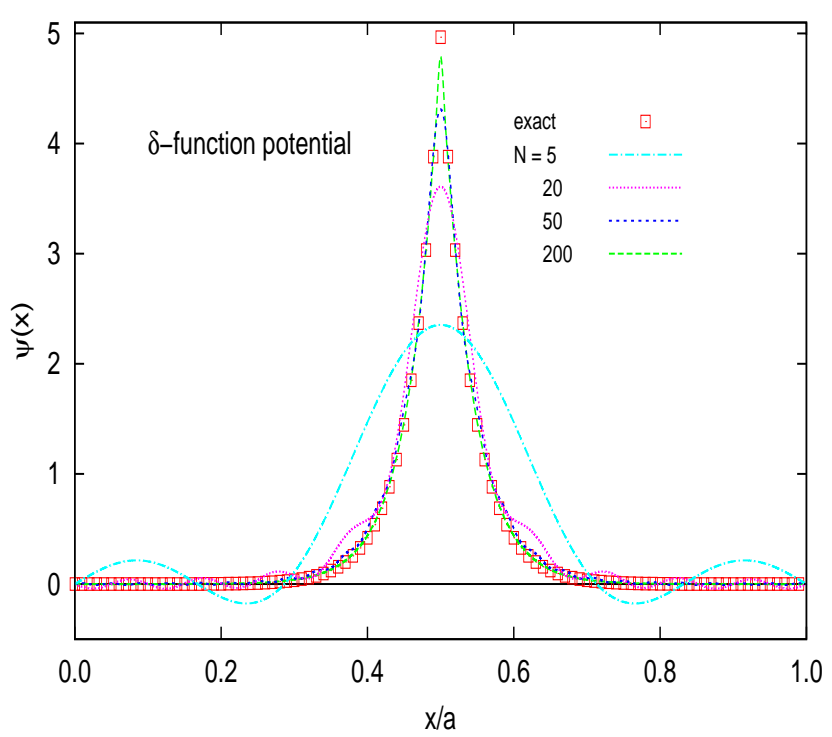

FIG. 5: (color online) Ground state energy wave function for a $\delta$-function potential, as calculated by diagonalization of matrices truncated at $N=5,20,50$, and 200. The exact result (see Appendix) is given by symbols. There is a cusp at the center, which requires smaller and smaller wavelength components to capture exactly. We used a $\delta$-function with strength $b V_{00}=-5 a E_{1}^{(0)}$, corresponding to the limiting case studied in Fig. 4

$b=a)$. Even the $3 \times 3$ matrix yields accurate ground state energy values all the way down to $b \approx 0.5 a$, and as the full frame of Fig. 4 (a) shows, finite $N$ results "peel off" as the width decreases. Note that the analytical result given by Eq. (18) differs from the numerical result near $b=a$ (see inset), because in this regime the two approaches solve different problems. The analytical result is for a finite square well of width $b$ with no background potential, and the numerical result includes the infinite square well in addition. ${ }^{14}$ Not surprisingly, the latter case reproduces the analytical shift noted earlier from $E_{0} / E_{1}^{(0)}=1$ to $E_{0} / E_{1}^{(0)}=1-5=-4$ (with $V_{00}=-5 E_{1}^{(0)}$ ).

Figure 4(b) clarifies the behavior in the very narrow well regime. Results for the delta function potential are given with symbols along the $b=0$ line. The lowest curve is given by the solution of a simple equation (see Eq. A2 in the Appendix). There is a considerable variation with the cutoff $N$ in the low $b / a$ regime. For example, even with a cutoff of $N=100$, meaning that unperturbed states with energy as high as 10000 are included, the result at $b=0$ is off from the $N \rightarrow \infty$ result by about $10 \%$. The reason is that as the finite well becomes narrower and the wave function becomes more confined, the latter contains components with very small (of order $b$ ) spatial variations. These can only be described by basis states with a similar strong variation, and such states are to be found only among the very high energy states of the infinite square well ${ }^{15}$ We use Eq. (13) and show in Fig. 5] the wave functions obtained numerically for the case of a delta function potential as we vary the cutoff of the size of the matrix retained. It is clear that for low cutoffs, only variations on a large length scale can be captured. Even for the highest cutoff used here, $N=200$, the numerical result still misses (though only slightly) the cusp at the centre of the figure. The same kind of plot can be examined for square well potentials of varying width, so that one can see directly that the matrix size required for convergence increases with narrower well width $b$ (as already seen through the energy in Fig. 4).

\section{SUMMARY}

The purpose of this paper is to provide some examples accessible to undergraduate students learning quantum mechanics for the first time that utilize matrix diagonalization. 16 Our goal is to provide a context within which a simple basis set can be used. The most straightforward case in one dimension is the infinite square well. We worked through some examples that are generally used in an introductory course on quantum mechanics, 1 with an emphasis on the harmonic oscillator. We showed that by embedding a (truncated) harmonic oscillator potential in an infinite square well, we can obtain extremely accurate solutions.

We also illustrated how to calculate wave functions and demonstrated that potentials with short length scales often require a large number of basis states for an accurate description.

The empowering aspect of this approach is that students can tackle bound state problems for any potential. For example, it is simple to find the low-lying states for a double well potential. The potential does not even have to be defined analytically 13

\section{Acknowledgments}

This work was supported in part by the Natural Sciences and Engineering Research Council of Canada (NSERC), by ICORE (Alberta), and by the Canadian Institute for Advanced Research (CIfAR). The author thanks Cindy Blois and James Day for suggestions to improve the presentation in the manuscript.

\section{APPENDIX A: DELTA FUNCTION POTENTIAL WELL}

Consider a delta function potential well with strength $\alpha=V_{0} b$ located at position $a_{0}$, inside an infinite square well between $x=0$ and $x=a$, that is,

$$
V(x)= \begin{cases}-b V_{0} \delta\left(x-a_{0}\right) & (0<x<a) \\ \infty & \text { otherwise }\end{cases}
$$


where $0<a_{0}<a$ (the symmetric case is for $a_{0}=a / 2$ ). The length scale $b$ is introduced to make contact with the finite square well potential discussed in Sec. III. The application of matching criteria yields the solution (we confine our discussion to the symmetric case only):

$$
\psi(x)=A \begin{cases}\sinh (\kappa x) / \sinh (\kappa a / 2) & (x<a / 2) \\ \sinh (\kappa(a-x)) / \sinh (\kappa a / 2) & (x>a / 2)\end{cases}
$$

The bound state energy $E$ is given by the solution of

$$
\tanh (\kappa a / 2)=\frac{2 \kappa}{\lambda}
$$

where $\kappa \equiv \sqrt{-\frac{2 m}{\hbar^{2}} E}$ and $\lambda \equiv-\frac{2 m}{\hbar^{2}} V_{0} b$. Equation (A2) must be solved numerically. The normalization constant is given by

$$
A=\sqrt{\frac{2}{a}} \frac{1}{\sqrt{1-\left(\frac{\lambda}{2 \kappa}\right)^{2}+\frac{\lambda}{\kappa^{2} a}}}
$$

The more familiar solution is obtained by allowing $a \rightarrow$ $\infty$, in which case $\kappa=\lambda / 2$, and a solution always exists. In this problem, with a finite infinite square well width $a$, a solution exists only if

A challenging exercise for students is to check how well Eq. (A4) holds for a finite square well of non-zero width $b$. Results for non-symmetrically placed delta functions can also be readily obtained, but are not as concise, and yield a condition for existence of a bound state which is more stringent than Eq. (A4).

1 D. J. Griffiths, Introduction to Quantum Mechanics (Pearson/Prentice Hall, Upper Saddle River, NJ, 2005), 2nd ed. There are many text books to choose from. I will cite this one as a typical, very good one.

2 J. M. Feagin, Quantum Mechanics with Mathematica (Springer, New York, 1994).

3 Bernd Thaller, Visual Quantum Mechanics (Springer, New York, 2000).

4 J. V. Kinderman, "A computing laboratory for introductory quantum mechanics," Am. J. Phys. 58, 568-573 (1990).

5 M. Belloni and W. Christian, "Time development in quantum mechanics using a reduced Hilbert space approach," Am. J. Phys. 76, 385-392 (2008).

6 E. Dagotto, "Correlated electrons in high-temperature superconductors," Rev. Mod. Phys. 66763 (1994).

7 J. L. Marin and S. A. Cruz, "On the harmonic oscillator inside an infinite potential well," Am. J. Phys. 56, 11341136 (1988).

8 M .T. Dove, Introduction to Lattice Dynamics (Cambridge University Press, Cambridge, 1993).

9 M. H. Anderson, J. R. Ensher, M. R. Matthews, C. E. Wieman, and E. A. Cornell, "Observation of Bose-Einstein condensation in a dilute atomic vapor," Science 269, 198201 (1995).

10 J .R. Schrieffer, Theory of Superconductivity (Benjamin/Cummings, Don Mills, 1983).

11 F. Marsiglio, "The spectral function of a one-dimensional Holstein polaron," Phys. Lett. A 180, 280-284 (1993); ibid., "Pairing in the Holstein model in the dilute limit," Physica C 244, 21-34 (1995).

12 W. H. Press, B. P. Flannery, S. A. Teukolsky, and W. T.
Vetterling, Numerical Recipes: The Art of Scientific Computing (Cambridge University Press, Cambridge, 1986).

13 At least one of my students simply programmed Matlab to evaluate Eq. (9) numerically for an arbitrary potential. Though not to my personal taste, many students find such a numerical evaluation much easier than slogging through analytical integrals. The numerical approach allows one to solve for bound states of any one-dimensional short range potential.

14 The analytical solution given by Eq. (18) provides the solution for a finite square well in an infinite space. Results will therefore differ from the numerical results as the width of the finite square well approaches the width of the infinite square well. The Appendix provides an analytical solution for the $\delta$-function potential in an infinite square well, which differs from the textbook solution of an infinite square well in infinite space.

15 These difficulties are well known. For example, bosons in a harmonic trap experience a short range boson-boson interaction. If we try to expand the many-particle wave function in terms of the eigenstates of the harmonic oscillator potential, convergence difficulties arise because of the disparity in length scales. Our problem illustrates this difficulty at the single particle level. I am indebted to Doerte Blume and Pierre-Nicholas Roy for discussions on this point.

16 Though often referred to as "Heisenberg matrix mechanics" it is now clear that Born should be credited with this technique. See, for example, Nancy Thorndike Greenspan, The End of the Certain World: The Life and Science of Max Born (Basic Books, Cambridge, MA, 2005). 